\section{Caracterização dos seminários por webconferência sobre saúde do adolescente e jovem da rede de núcleos de telessaúde de Pernambuco}

\section{A characterization of web-conference seminars on the health of adolescents and young people at cyber-health centers in the Brazilian State of Pernambuco}

Elisabeth Lima Dias da Cruz 1 Magdala de Araújo Novaes 2 Josiane Lemos Machiavelli 3 Valdenice Aparecida de Menezes 4

\begin{abstract}
Objectives: to characterize the profile of webconference seminars on the health of adolescents and young people using registers from the cyber-education service of the Pernambuco Network of CyberHealth Units (RedeNUTES).

Methods: a quantitative, retrospective, descriptive, exploratory study was carried out with dada from 1348 occasions on which health workers participated in seminars in the year 2009.

Results: RedeNUTES were in existence in $45 \%$ of municipalities in Pernambuco. 148 seminars were held (19\% in the field of the health of adolescents and young people) with the participation of all the Regional Health Management Teams; with an especially strong participation on the part of nursing (51\%) and dental health teams (16.5\%). During the exchange of experiences, 151 questions were answered (an average of 5.4 per seminar), with strong participations of $55.5 \%$ and $19.7 \%$ from the aforementioned groups.

Conclusions: is a need to encourage the participation of health workers in on-going training to strengthen continuous health promotion and all-round care for adolescents.
\end{abstract}

Key words Education, distance, Telemedicine, Primary health care, Adolescent health
1-3 Núcleo de Telessaúde de Pernambuco. Universidade Federal de Pernambuco. Av. Prof. Moraes Rego. s.n. Cidade Universitária. Recife, PE, Brasil. CEP: 50.670-420. E-mail: elisabeth.cruz@gmail.com 4 Faculdade de Odontologia de Pernambuco. Universidade de Pernambuco. Camaragibe, PE, Brasil.

\section{Resumo}

Objetivos: caracterizar o perfil dos seminários por webconferência sobre saúde do adolescente e jovem, a partir dos registros do serviço de teleeducação da Rede de Núcleos de Telessaúde (RedeNUTES) de Pernambuco.

Métodos: estudo retrospectivo, descritivo e exploratório, de abordagem quantitativa, com dados de 1348 participações de profissionais de saúde nos seminários ocorridos no ano de 2009.

Resultados: a RedeNUTES esteve presente em 45\% dos municípios de Pernambuco. Foram realizados 148 seminários (19\% - saúde do adolescente e jovem) com participação de todas as Gerências Regionais de Saúde; destacando a participação das equipes de enfermagem (51\%) e saúde bucal (16,5\%). No momento de troca de experiências foram feitos 151 esclarecimentos de dúvidas (média de 5,4 dúvidas por seminário), destacando as mesmas equipes em $55,5 \%$ e $19,7 \%$, respectivamente.

Conclusões: existe uma necessidade de estimular a participação dos profissionais de saúde nas capacitações permanentes para fortalecer as ações de promoção da saúde e o cuidado integral de forma contínua ao grupo dos adolescentes.

Palavras-chave Educação à distância, Telemedicina, Atenção primária à saúde, Saúde do adolescente 


\section{Introdução}

A Educação à Distância (EAD) é uma modalidade educacional na qual a mediação didático-pedagógica nos processos de ensino-aprendizagem ocorre com a utilização de meios e tecnologias de informação e comunicação, desenvolvendo atividades educativas em lugares ou tempos diversos. 1,2

Define-se também a EAD como um modelo educativo organizado, em que grandes grupos educacionais e/ou profissionais desempenham o papel de sujeito, (re)construindo o conhecimento, de forma interativa e diversificada, possibilitando a troca de experiências profissionais e de vida, por meio de um aprendizado flexível e independente. 3-5

Para que esse processo educacional ocorra são empregados diversos modelos de EAD provenientes de combinações de linguagens e recursos tecnológicos, os quais podem ser utilizados juntamente ao material impresso. Os recursos tecnológicos encontrados para esta finalidade são a transmissão em televisão com as fitas de vídeo e o áudio, o CD-ROM, os kits de material experimental, a orientação por computador, as videoconferências, as teleconferências, o correio eletrônico, o fórum, o chat, as orientações presenciais e por correspondência.3,6-8

De acordo com as etapas evolutivas da EAD é visível a modificação da nomenclatura e da utilização desses recursos. O que é chamado de teleeducação já foi considerada como tele-ensino, ensino à distância, estudos externos, estudo independente, estudo em casa, educação por correspondência ou estudo por correspondência.3,4,9

Observando as dificuldades enfrentadas por profissionais de saúde que atuam na Estratégia Saúde da Família (ESF), nas mais distantes regiões geográficas dos Estados Brasileiros, e consequentemente, afastadas dos centros de referência acadêmica, a EAD pode ser considerada uma estratégia para vencer essas barreiras, tanto na Educação Permanente em Serviço (EPS) como também no apoio ao processo de trabalho frente às novas tecnologias. 6,10

Esse processo educativo à distância facilita a realização das atividades de contínua capacitação do profissional sem afastá-lo, por muito tempo, de suas atividades diárias nas Unidades de Saúde da Família (USF), pois poderá ocorrer por meio da teleconferência, videoconferência ou webconferência, com total interatividade, bem como disponibilizando um arsenal de recursos multimídia. 6,11

Visando à qualificação da atenção à saúde e à educação para o trabalho, para fortalecer as ativi- dades de prevenção, promoção, recuperação e manutenção à saúde, o Ministério da Saúde, em parceria com os Ministérios de Ciência e Tecnologia e Educação, implantou, em 2007, o Programa Telessaúde Brasil.12,13

Este Programa iniciou-se com o Projeto Piloto Nacional de Telessaúde aplicado à atenção primária, o qual foi desenvolvido em nove Estados: Amazonas, Ceará, Pernambuco, Goiás, Minas Gerais, Rio de Janeiro, São Paulo, Santa Catarina e Rio Grande do Sul. Esses Estados foram escolhidos para contemplar as cinco regiões do país e por já apresentarem experiência na área de telessaúde.

Em 2010, o Programa Telessaúde Brasil passou a apoiar a Estratégia de Saúde da Família no Sistema Único de Saúde, por meio da Portaria n ${ }^{\circ} 402$, de 24 de fevereiro de 2010, expandindo assim os serviços de telessaúde existentes e implantando novos núcleos de telessaúde nos demais Estados Brasileiros. 14

Em Pernambuco, o programa é coordenado e executado pelo Núcleo de Telessaúde (NUTES), localizado na Universidade Federal de Pernambuco (UFPE), o qual possibilitou a expansão da Rede de Núcleos de Telessaúde (RedeNUTES), criada em 2003. Em dezembro de 2009, a RedeNUTES possuía 98 pontos de telessaúde nas USFs, beneficiando 83 municípios, distribuídos nas 11 Gerências Regionais de Saúde (GERES). 15,16

A RedeNUTES oferta, por meio da internet, os serviços de tele-educação e teleassistência. Em 2009, na área de tele-educação, foram oferecidos os seminários distribuídos em quatro grandes áreas temáticas: saúde da criança e do adolescente; saúde mental; enfermagem; e temas gerais em saúde coletiva. Além disso, as equipes de saúde também dispunham do Ambiente Virtual de Aprendizagem da RedeNUTES (AVA RedeNUTES), que funcionava como um repositório de conteúdos produzidos a partir dos seminários (vídeos, apresentações eletrônicas, dentre outros). 16

Dentro deste contexto, o presente trabalho teve como objetivo caracterizar o perfil dos seminários por webconferência sobre saúde do adolescente e jovem, a partir dos dados do serviço de teleeducação da RedeNUTES de Pernambuco.

\section{Métodos}

Estudo restrospectivo, descritivo e exploratório, a partir de informações das bases de dados do serviço de tele-educação da RedeNUTES. Para a coleta de dados, os profissionais participantes dos seminários foram comunicados sobre os objetivos das infor- 
mações coletadas durante as sessões e que ao término da apresentação teriam que acessar o ambiente virtual de aprendizagem para o preenchimento on line do Termo de Consentimento Livre $\mathrm{e}$ Esclarecido e do instrumento de coleta de dados.

Vale ressaltar que a atividade de preenchimento do instrumento de coleta estava vinculada à aceitação do profissional em participar da pesquisa. Caso não concordasse com os termos, o profissional clicava em "cancelar", não visualizando, assim, o instrumento de pesquisa.

A população do estudo foi composta por 1348 profissionais das Unidades de Saúde da Família do Estado de Pernambuco que participaram dos seminários sobre saúde do adolescente e jovem entre janeiro e dezembro de 2009.

Os dados foram extraídos de planilha excel e analisados no software SPSS (Statistical Package for the Social Sciences) versão 13 (análise descritiva) e no software estatístico R versão 2.11 (análise estatística).

Os resultados foram analisados por meio de técnicas estatísticas descritivas através de distribuições absolutas e percentuais, e testes estatísticos de qui-quadrado de homogeneidade, utilizando o nível de significância de $5 \%$ e os intervalos de confiabilidade de $95 \%$.

Foram consideradas de interesse para o estudo as variáveis: áreas temáticas dos seminários, participação dos profissionais de saúde nos temas sobre saúde do adolescente e jovem, participação por categoria profissional, cobertura da RedeNUTES em Pernambuco e participação das GERES nesses seminários.

O projeto foi aprovado pela Comissão de Ética em Pesquisa da Universidade de Pernambuco, sob o
CAAE: 0114.0.172.097-10.

\section{Resultados}

Os seminários foram realizados com a utilização do software adobe connect e ocorreram semanalmente com duração média de uma hora, sendo trinta minutos para apresentação do tema e trinta minutos para interação entre os participantes conectados e o apresentador para esclarecimento das dúvidas referentes ao tema ministrado, seja dúvida clínica ou relacionada ao processo de trabalho.

Por meio do AVA RedeNUTES foram disponibilizados as apresentações, os vídeos e os materiais complementares referentes aos conteúdos abordados, assim como o questionário de avaliação qualitativa dos seminários.

Dos 148 seminários realizados, $19 \%$ apresentaram temas voltados para saúde do adolescente e jovem, sendo distribuídos nas seguintes proporções nas áreas temáticas: saúde da criança e do adolescente $(50 \%)$, saúde mental (32\%), temas gerais em saúde coletiva (14\%) e enfermagem (4\%), que apresentaram diferenças estatisticamente significantes $(p<0,001)$.

$\mathrm{Na}$ Tabela 1 estão apresentados a distribuição dos participantes por temas ministrados sobre saúde do adolescente e jovem nos seminários.

Observou-se que esses seminários tiveram como foco principal os assuntos relacionados ao crescimento e desenvolvimento do adolescente, com $39,6 \%$ dos seminários ministrados, seguidos dos temas de saúde mental infanto-juvenil e saúde bucal correspondendo a $25 \%$ e $14,3 \%$, respectivamente.

Foram ministrados os seguintes conteúdos: imunização; infecções respiratórias; otites;

Tabela 1

Distribuição dos participantes nos temas ministrados sobre saúde do adolescente e jovem nos seminários por webconferência. Pernambuco; 2009.

\begin{tabular}{lcc}
\hline Temas & N & $\%$ \\
\hline Crescimento e desenvolvimento do adolescente & 534 & 39,6 \\
Saúde mental: infanto-juvenil & 338 & 25,0 \\
Saúde bucal & 193 & 14,3 \\
Sexualidade na adolescência & 101 & 7,5 \\
Saúde coletiva & 59 & 4,4 \\
Violência & 44 & 3,3 \\
Pediatria & 40 & 3,0 \\
Suicídio & 39 & 2,9 \\
Total & 1348 & 100,0 \\
\hline
\end{tabular}

Fonte: RedeNUTES/PE - Registro de participação dos profissionais nos seminários por webconferência; 2009. 
enteroparasitoses; amigdalite estreptocócica; autismo infantil; hiperatividade; emergências em odontopediatria; obesidade infantil; atenção à saúde infanto-juvenil: antecedentes históricos e cenário atual; anorexia e bulemia; desenvolvimento puberal precoce e tardio; sexualidade na adolescência; gravidez na adolescência; adolescência e o uso de drogas; prematuramente mãe de bebê prematuro; DST na adolescência; tuberculose pulmonar na infância e adolescência; violência na adolescência; doenças sexualmente transmissíveis (DST)/ síndrome da imunodeficiência adquirida (Aids); problemas andrológicos mais comuns na adolescência.

Entre os assuntos ministrados, o seminário intitulado "DST/Aids" obteve destaque em participação dos profissionais de saúde, apresentando o maior número de pontos conectados, ou seja, $25 \%$ das USFs parceiras da RedeNUTES.

Na Tabela 2 verifica-se a distribuição das categorias profissionais participantes dos seminários e esclarecimentos das dúvidas sobre a temática ministrada. Nestas informações, foi preciso realizar um decréscimo de 0,2 nas participações da categoria profissional de Agente Comunitário de Saúde (ACS), pois em cada USF há uma média de cinco ACS para cada profissional de outra categoria.

Nos seminários houve destaque em participação para os profissionais da equipe de enfermagem (enfermeiros e técnicos de enfermagem - 51\%), seguidos pela equipe de saúde bucal (cirurgiõesdentistas, atendentes e técnicos em saúde bucal $16,5 \%)$; outras categorias profissionais $(13,2 \%)$ e agentes comunitários de saúde (11\%)

$\mathrm{Na}$ interação entre os participantes conectados e o apresentador para esclarecimento de dúvidas referentes ao tema ministrado, seja questão clínica ou relacionada ao processo de trabalho, foram feitos 151 questionamentos, o que corresponde a uma média de 5,4 dúvidas por seminário.

Ao se aplicar a correção de proporção entre as categorias profissionais, manteve-se a equipe de enfermagem $(55,5 \%)$ e a equipe de saúde bucal $(19,7 \%)$ como destaque em participação do momento de troca de experiências. No entanto, há uma inversão entre os agentes comunitários de saúde $(7,7 \%)$ e outras categorias profissionais $(6 \%)$.

$\mathrm{Na}$ Tabela 3 apresenta-se a cobertura da RedeNUTES em Pernambuco, presente em $45 \%$ dos municípios do Estado durante o ano de 2009.

Na Tabela 4, observa-se que $100 \%$ das Gerências Regionais de Saúde participaram dos seminários e do momento de interação com o palestrante para esclarecimento de dúvidas, destacando-se em maior participação as GERES VI, IV, V, XI, I.

Em relação ao quantitativo de profissionais que assistiram aos seminários, houve um total de 1348 profissionais de saúde beneficiados diretamente, obtendo a média de 48 participantes por sessão.

Vale ressaltar que dos 98 pontos de telessaúde, $82 \%$ estiveram conectados às atividades de educação permanente na temática saúde do adolescente e jovem, apresentando uma média de 12 pontos conectados por sessão de webconferência.

Tabela 2

Distribuição das categorias profissionais participantes dos seminários por webconferência e esclarecimento de dúvidas em saúde do adolescente e jovem. Pernambuco; 2009.

\begin{tabular}{|c|c|c|c|c|c|c|}
\hline \multirow[t]{2}{*}{ Categoria profissional } & \multicolumn{3}{|c|}{ Seminários por webconferência } & \multicolumn{3}{|c|}{ Esclarecimento de dúvidas } \\
\hline & $\mathrm{n}$ & $\%$ & $p$ & $\mathrm{n}$ & $\%$ & $p$ \\
\hline Médico & 58 & 6,2 & $<0.001$ & 3 & 2,6 & $<0.001$ \\
\hline Enfermeiro & 269 & 28,8 & & 41 & 35,0 & \\
\hline Técnico em enfermagem & 207 & 22,2 & & 24 & 20,5 & \\
\hline Cirurgião-dentista & 79 & 8,5 & & 16 & 13,7 & \\
\hline Atendente de saúde bucal & 70 & 7,5 & & 6 & 5,1 & \\
\hline Técnico em saúde bucal & 5 & 0,5 & & 1 & 0,9 & \\
\hline Agente comunitário de saúde & $103 *$ & $11,0 *$ & & 9 * & 7,7 * & \\
\hline Psicólogo & 10 & 1,1 & & 8 & 6,8 & \\
\hline Assistente social & 5 & 0,5 & & - & - & \\
\hline Educador físico & 4 & 0,4 & & - & - & \\
\hline Terapeuta ocupacional & 1 & 0,1 & & 2 & 1,7 & \\
\hline Outras categorias profissionais & 123 & 13,2 & & 7 & 6,0 & \\
\hline Total & 934 & 100,0 & & 117 & 100,0 & \\
\hline
\end{tabular}

* Abatimento de 0,2 - utilizado para correção de proporção entre as categorias profissionais na Unidade de Saúde da Família. Fonte: RedeNUTES/PE - Registro de participações dos profissionais nos seminários por webconferência; 2009. 
Tabela 3

Distribuição dos municípios parceiros da RedeNUTES segundo as Gerências Estaduais de Saúde (GERES). Pernambuco; 2009.

\begin{tabular}{lcrc}
\hline \multirow{2}{*}{ GERES } & Municípios & \multicolumn{2}{c}{ Municípios parceiros } \\
\cline { 3 - 4 } & & $n$ & $\%$ \\
\hline I & & & 63,2 \\
II & 19 & 12 & 29,0 \\
III & 31 & 9 & 45,5 \\
IV & 22 & 10 & 37,5 \\
V & 32 & 12 & 19,0 \\
VI & 21 & 4 & 61,5 \\
VII & 13 & 8 & 57,1 \\
VIII & 7 & 4 & 71,4 \\
IX & 7 & 5 & 54,5 \\
X & 11 & 6 & 41,7 \\
XI & 12 & 5 & 80,0 \\
Total & 10 & 8 & 44,9 \\
\hline
\end{tabular}

Fonte: RedeNUTES/PE - Planilha de acompanhamento; 2009.

\section{Tabela 4}

Participação profissional dos municípios parceiros, segundo distribuições por Gerências Estaduais de Saúde (GERES), nos seminários por webconferência e esclarecimento de dúvidas em saúde do adolescente e jovem. Pernambuco; 2009.

\begin{tabular}{lrrrr}
\hline \multirow{2}{*}{ GERES } & \multicolumn{2}{c}{ Participação profissional por GERES } & \multicolumn{2}{c}{ Esclarecimento de dúvidas } \\
\cline { 2 - 5 } & $\mathrm{n}$ & $\%$ & $p$ & $\mathrm{n}$ \\
\hline I & 178 & 13,2 & 28 & 18,5 \\
II & 50 & 3,7 & 6 & 4,0 \\
III & 18 & 1,3 & 3 & 2,0 \\
IV & 225 & 16,7 & 9 & 6,0 \\
V & 200 & 14,8 & 16 & 10,6 \\
VI & 256 & 19,0 & 43 & 28,5 \\
VII & 94 & 7,0 & 9 & 6,0 \\
VIII & 47 & 3,5 & 4 & 2,6 \\
IX & 14 & 1,0 & 1 & 0,6 \\
X & 74 & 5,5 & 6 & 4,0 \\
XI & 192 & 14,3 & 26 & 17,2 \\
Total & 1348 & 100,0 & 151 & 100,0
\end{tabular}

Fonte: RedeNUTES/PE - Planilha de acompanhamento; 2009. 


\section{Discussão}

A educação permanente em serviço é uma das estratégias que possibilita construir um novo estilo de formação do profissional. É a aprendizagem no trabalho, em que o aprender e o ensinar se incorporam ao cotidiano das organizações e ao trabalho, proporcionando a troca de experiência entre profissionais, confrontando as práticas locais de cada região e o conhecimento científico a partir da problematização do processo de trabalho. Isso fortalece a reorganização dos serviços públicos, pois propicia a elaboração de estratégias para enfrentar os obstáculos de consolidação do Sistema Único de Saúde (SUS) no campo de gestão, de formação e de controle social.17-20

Na RedeNUTES de Pernambuco, as atividades de educação permanente acontecem na modalidade a distância, por meio dos seminários. Sua aplicabilidade permite atualizações em saúde da família de forma contínua no local de trabalho - as Unidades de Saúde da Família - e no horário de expediente, sem que os profissionais precisem interromper as atividades, por dias ou semanas, para participarem de atividades de capacitação. ${ }^{21}$

Em 2009, Pernambuco contava com o total de 16.345 profissionais de saúde da família (médico, enfermeiro, cirurgião-dentista, técnico de enfermagem, ACS). 22 É importante ter em conta que $45 \%$ destes profissionais encontravam-se em atividades nas USF dos municípios parceiros da RedeNUTES e que, dentre esses, $15 \%$ foram beneficiados diretamente pelo programa, uma vez que estavam alocados nas USFs que receberam o ponto de telessaúde.

Os benefícios aos profissionais de saúde que participam das atividades de tele-educação são inúmeros, além das capacitações permanentes. Há o acesso aos protocolos sistematizados, à pesquisa colaborativa entre os centros de ensino e às sessões de teleconsultorias, em que eles possuem acesso direto ao apresentador para questionar sobre conteúdos e/ou condutas de saúde.7,23,24

Outro benefício é a possibilidade de construir o seu próprio conhecimento por meio de trocas de experiências com profissionais de outras regiões, os quais possuem uma realidade diferenciada para a execução de uma mesma atividade. $\mathrm{O}$ acesso aos materiais das capacitações que são disponibilizados no AVA RedeNUTES (vídeos, apresentações e materiais complementares do conteúdo apresentado) também merece destaque, pois pode ser acessado de qualquer computador e em qualquer horário. 24

Dentre as áreas temáticas abordadas nos seminários (saúde da criança e do adolescente; saúde mental; enfermagem; e temas gerais em saúde coletiva), a única que ocorria no turno da manhã era a área de saúde da criança e do adolescente sendo importante notar que, conforme estudo similar, as atividades de tele-educação possuem maior participação no turno da tarde. ${ }^{23}$ Isso ocorre devido à grande demanda de atendimento ao usuário no turno da manhã, principalmente nas USFs do interior do Estado.

Dessa forma, apesar de a RedeNUTES dispor de um horário específico para discutir os temas sobre saúde da criança e do adolescente nos seminários, para as realizações das capacitações permanentes, estas foram distribuídas nas quatro áreas temáticas, ou seja, nos dois turnos. Isso favoreceu a participação dos profissionais de saúde e a reorganização de suas atividades diárias na USF.

Nesse processo de aperfeiçoamento permanente, é recomendável considerar temas específicos sobre o adolescente para que os profissionais de saúde realizem de forma adequada as ações de promoção da saúde, prevenção de agravos, assistência e reabilitação para este grupo etário. 25-27

Os temas ministrados nos seminários contemplaram as seguintes áreas prioritárias do Programa Saúde do Adolescente: projeto de vida, sonhos e autoestima; participação juvenil; crescimento e desenvolvimento; relacionamentos sociais; direitos sexuais e reprodutivos; sexualidade; álcool e outras drogas; violência doméstica e social; e saúde bucal.25,27-29

Quanto à maior participação dos profissionais de saúde no seminário intitulado "DST/Aids", observase que esta temática encontra-se em destaque nos protocolos nacionais, estaduais e municipais de Doenças Sexualmente Transmissíveis, assim como entre as ações de promoção da saúde e prevenção dos agravos relacionados aos aspectos de vulnerabilidade dos adolescentes. Por esta razão, são importantes as iniciativas de capacitações específicas orientadas ao trabalho interdisciplinar para melhorar o atendimento a esse público. $25,27-29$

O levantamento realizado no presente estudo demonstrou que é possível realizar caracterizações por temas prioritários do Ministério da Saúde, a fim de aprimorar as atividades de educação, para os profissionais de saúde, em áreas importantes para atenção primária à saúde, porém de pouca prioridade em sua execução, devido aos baixos números no quadro de morbi-mortalidade e busca da atenção à saúde pelos adolescentes.

Neste sentido, recomenda-se que para as atividades dos seminários se adotem metodologias interativas e articuladas com o processo de trabalho, 
buscando parcerias inter-setoriais para o fortalecimento da saúde integral do adolescente de forma contínua.

Quanto ao perfil dos profissionais participantes do processo educacional à distância em saúde do adolescente e jovem, observou-se que a equipe de enfermagem teve uma maior adesão, provavelmente por estar a mais tempo na USF, ou por já ter experiências anteriores com educação à distância.26,30

Em relação aos demais profissionais, a participação da categoria médica encontra-se pouco expressiva. Isso, provavelmente, está relacionado à alta rotatividade desse profissional nas USFs, e ao fato de muitos possuírem dois ou mais vínculos empregatícios, o que reduz seu tempo de permanência na unidade de saúde. Quanto à equipe de saúde bucal, pode-se afirmar que a participação foi satisfatória nos seminários de saúde do adolescente e do jovem, apesar dessa equipe encontrar-se, em geral, executando atividades para duas equipes de saúde da família. 6

É importante ressaltar que a instalação dos pontos de telessaúde nas USFs provocou alteração na rotina diária das equipes de saúde, não acostumados com o uso do computador para educação permanente no ambiente de trabalho, havendo a necessidade de adaptação de alguns profissionais aos equipamentos tecnológicos e ao processo educativo à distância.6,31

Para o fortalecimento das ações locais de telessaúde é necessário: consolidar o apoio político, tanto no setor da saúde como na educação; envolver os demais profissionais da estratégia saúde da família e do Núcleo de Apoio a Saúde da Família (NASF) nas capacitações em saúde do adolescente e jovem, já que esse público deve ser acompanhado de forma multiprofissional; desenvolver integração das atividades de tele-educação a outros programas que envolvam os adolescentes e jovens como público alvo; estimular os profissionais de saúde a interagir com as atividades educativas no âmbito escolar, pois esse local é o que possui maior concentração dessa população em grupos; estimular a utilização do

\section{Referências}

1. Litto FM, Formiga MMM, orgs. Educação a Distância: o estado da arte. São Paulo: Pearson Education do Brasil; 2009 .

2. Nogueira VO, Marin HF, Cunha ICKO. Informações online sobre transporte intra-hospitalar de pacientes críticos adultos. Acta Paul Enferm. 2005; 18: 390-6. material disponibilizado no AVA RedeNUTES para a realização das atividades de promoção à saúde do adolescente e jovem; divulgar mais as ações desenvolvidas e seus principais indicadores, demonstrando, assim, o potencial do serviço e estimulando outros profissionais a integrarem a rede. Em resumo os resultados apresentados neste trabalho mostraram que, em 2009, a caracterização dos seminários sobre saúde do adolescente e jovem apresentou-se da seguinte forma: a) as atividades de educação permanente em saúde da criança e do adolescente obtiveram uma maior frequência quando comparadas às áreas de saúde mental, temas gerais e enfermagem; b) houve maior participação dos profissionais nos temas ministrados sobre crescimento e desenvolvimento do adolescente, saúde mental infanto-juvenil e saúde bucal; c) as equipes de enfermagem e saúde bucal se destacaram em participação nos seminários e no momento de inte-ração para esclarecimentos de dúvidas clínicas ou de processo de trabalho d) a cobertura da RedeNUTES é de $45 \%$ dos municípios do Estado de Pernambuco; e) todas as Gerências Regionais de Saúde participaram desse processo educativo.

Em conclusão a presente pesquisa indica que, em uma área temática específica, é possível realizar estudos referentes às demais áreas temáticas, assim como existe a necessidade de estimular a participação dos profissionais de saúde nas capacitações permanentes para fortalecer as ações de promoção da saúde e o cuidado integral de forma contínua aos adolescentes e jovens dos municípios parceiros da Rede de Núcleos de Telessaúde de Pernambuco.

\section{Agradecimentos}

À Coordenação e vice-coordenação do Núcleo de Telessaúde da UFPE por permitir e apoiar o desenvolvimento da pesquisa no estabelecimento. À Equipe da RedeNUTES por apoiar o desenvolvimento dessa pesquisa.

3. Belloni ML. Ensaio sobre a educação a distância no Brasil. Rev Educ Soc. 202; 78: 117-42.

4. Sanavria CV. A avaliação da aprendizagem na educação a distância: concepções e práticas de professores de ensino superior [dissertação]. Campo Grande: Universidade Católica Dom Bosco; 2008. 
5. Quelhas MCF, Lopes MHBM, Ropoli EA. Educação à distância em processos de esterilização de materiais. Rev Esc Enferm USP. 2008; 42: 697-705.

6. Oliveira MAN. Educação à Distância como estratégia para a educação permanente em saúde: possibilidades e desafios. Rev Bras Enferm. 2007; 60: 585-9.

7. Forsetlund L, Bjørndal A, Rashidian A, Jamtvedt G, O'Brien MA, Wolf F, Davis D, Odgaard-Jensen J, Oxman AD Continuing education meetings and workshops: effects on professional practice and health care outcomes. Cochrane Database of Systematic Reviews; 2009, Issue 2.

8. International Telecommunication Union. New technologies for rural applications. Final Report of ITU-D Focus Group 7. Bridging the digital divide, providing digital opportunities for all; 2000

9. Barilli ECVC. Aplicação de métodos e técnicas de realidade virtual para apoiar processos educativos a distância que exijam o desenvolvimento de habilidades motoras: um ambiente virtual para a aprendizagem dos procedimentos antropométricos ligados ao Sistema de Vigilância Alimentar e Nutricional do Sistema de Saúde Brasileiro [tese]. Rio de Janeiro: Universidade Federal do Rio de Janeiro; 2007.

10. Maftum MA, Campos JB. Capacitação pedagógica na modalidade de educação a distância: desafio para ativar processos de mudança na formação de profissionais de saúde. Cogitare Enferm. 2008; 13: 132-9.

11. Camacho ACL. Educação a distância na disciplina de legislação, ética e exercício de enfermagem. Rev Bras Enferm. 2009; 62: 151-5.

12. Brasil. Ministério da Saúde. Portaria $n^{\circ} 35$ de 04 de janeiro de 2007. Institui, no âmbito do Ministério da Saúde, o Programa Nacional de Telessaúde. Brasília, DF; 2007.

13. Garcia RM, Baptista R. Educação a distância para a qualificação dos profissionais do SUS: perspectivas e desafios. Rev Baiana Saúde Pública. 2007; 31: 70-8

14. Brasil. Ministério da Saúde. Portaria $n^{0} 402$ de 24 de fevereiro de 2010. Institui, em âmbito nacional, o Programa Telessaúde Brasil para apoio à Estratégia de Saúde da Família no Sistema Único de Saúde. Brasília, DF; 2010.

15. Brasil. Ministério da Saúde. Programa Nacional de Telessaúde na Atenção Primária à Saúde. Sobre o programa [Internet]. 2009. [acesso em 26 Jul 2009]. Disponível em http://www.telessaudebrasil.org.br/php/level.php?lang=pt\& component $=42$ \&item $=1$

16. Rede de Núcleos de Telessaúde de Pernambuco [Internet]. 2010. [acesso em 15 jan 2010]. Disponível em http://www.redenutes-pe.ufpe.br/

17. Brasil. Ministério da Saúde. Política Nacional de Educação Permanente em Saúde. Série B Textos Básicos de Saúde. Série Pacto pela Saúde, v.9. Brasília, DF; 2009.

18. Lopes SRS, Piovesan ETA, Melo LO, Pereira MF Potencialidades da educação permanente para a transformação das práticas de saúde. Rev Com Ciências Saúde. 2007; 18: 147-55. Disponível em: http://www.fepecs. edu.br/revista/Vol18_2art06.pdf

Recebido em 3 de outubro de 2011

Versão final apresentada em 30 de janeiro de 2012

Aprovado em 10 de fevereiro de 2012
19. Moimaz SAS, Rocha NB, Rovida TAS, Garbin CAS, Garbin AJI. A re/organização dos serviços de saúde por meio da Educação Permanente. Odontol Soc. 2009; 11: 1-7.

20. Pereira LA, Monteiro FA. Boletim Informativo/eletrônico, Rede Unida, $\mathrm{n}^{\circ}$ 17, 23 fev 2004. [acesso em 22 fev 2009]. Disponível em: http://www.redeunida.org.br/educacaoprofissional/boletim-inrede-1/boletim-inrede/

21. Susan AZ, Michael GK, Zak H, Louis GC, Douglas SW. Tele-education in a telemedicine environment: implications for rural health care and Academic Medical Centers. J Med Syst. 1999; 23: 107-22.

22. SES-PE (Secretaria de Saúde do Estado de Pernambuco). Planilha de repasse financeiro da Atenção Primária do Estado de Pernambuco; 2009.

23. Lima CMAO, Monteiro AMV, Ribeiro EB, Portugal SM, Silva LSX, João Jr M. Videoconferências: sistematização e experiências em telemedicina. Radiol Bras. 2007; 40: 3414.

24. Fahey A, Gelber H. Tele-education: a collaborative project in the delivery of mental health education in rural Victoria. Bendigo Health Care Group Psychiatric Services and Royal Children's Hospital Mental Health Service (MHSKY); 2000.

25. Ruzany MH. Mapa de situação de saúde do adolescente no município do Rio de Janeiro [tese]. Rio de Janeiro: Escola Nacional de Saúde Pública da Fundação Oswaldo Cruz; 2000

26. Germano RM, Formiga JMM, Melo MNB, Vilar RLA, Almeida Jr JJ. Capacitação das Equipes do PSF: desvendando uma realidade. Rio Grande do Norte, Natal; 2005. [acesso em 15 jan 2011] Disponível em: http://www.observarh.org.br/observarh/repertorio/Repertorio_ObservaRH/N ESC-RN/Capacitacao equipes PSF.pdf

27. São Paulo. Secretaria da Saúde. $2^{\circ}$ caderno de apoio ao acolhimento a saúde da família: orientações, rotinas e fluxos sob a ótica do risco/vulnerabilidade. São Paulo; 2004

28. São Paulo. Secretaria da Saúde. Manual de atenção à saúde do adolescente. Coordenação de Desenvolvimento de Programas e Políticas de Saúde (CODEPPS). São Paulo; 2006

29. Brasil. Ministério da Saúde. Saúde integral de adolescentes e jovens: orientações para a organização de serviços de saúde à Saúde. Série A. Normas e Manuais Técnicos. Brasília, DF; 2005.

30. Formiga JMM, Germano RM, Timoteo RPS, Vilar RLA, Teodosio SSS, Dantas SMM. Encurtando distâncias, vencendo obstáculos, articulando saberes: uma experiência em enfermagem. [acesso em 15 jan 2011]. Disponível em: http://www.observatorio.nesc.ufrn.br/texto_relato06.pdf.

31. Cruz ELD, Sanches LMP, Alves DS, Machiavelli J, Novaes M. A Tele-enfermagem como ferramenta para capacitação do enfermeiro que atua no Programa Saúde da Família. In: IV Congresso Brasileiro de Telemedicina e Telessaúde; 2009; Belo Horizonte, MG. 